\title{
Value of neutrophil-to-lymphocyte ratio for predicting lung cancer prognosis: A meta-analysis of 7,219 patients
}

\author{
YU YU, LEI QIAN and JIUWEI CUI \\ Cancer Center, The First Hospital of Jilin University, Changchun, Jilin 130021, P.R. China
}

Received February 24, 2017; Accepted July 18, 2017

DOI: $10.3892 /$ mco.2017.1342

\begin{abstract}
Current evidence suggests that the neutrophil-to-lymphocyte ratio (NLR) may be a biomarker for poor prognosis in lung cancer, although this association remains controversial. Therefore, a meta-analysis was performed to evaluate the association between NLR and lung cancer outcome. A systematic literature search was performed through the PubMed, Embase and Cochrane Library databases (until July 30, 2016), to identify studies evaluating the association between NLR and overall survival (OS) and/or progression-free survival (PFS) among patients with lung cancer. Based on the results of this search, data from 18 studies involving 7,219 patients with lung cancer were evaluated. The pooled hazard ratio (HR) suggested that elevated pretreatment NLR predicted poor OS $[\mathrm{HR}=1.46,95 \%$ confidence interval (CI): 1.30-1.64] and poor PFS (HR=1.42, 95\% CI: 1.15-1.75) among patients with lung cancer. Subgroup analysis revealed that the prognostic value of NLR for predicting poor OS increased among patients who underwent surgery $(\mathrm{HR}=1.50,95 \% \mathrm{CI}: 1.21-1.84)$ or patients with early-stage disease $(\mathrm{HR}=1.64,95 \% \mathrm{CI}: 1.37-1.97)$. An NLR cut-off value of $\geq 4$ significantly predicted poor $\mathrm{OS}$ ( $\mathrm{HR}=1.56,95 \% \mathrm{CI}$ : 1.31-1.85) and PFS (HR=1.54, 95\% CI: 1.13-1.82), particularly in the cases of small-cell lung cancer. Thus, the results of the present meta-analysis suggested that an elevated pretreatment NLR (e.g., 24) may be considered as a biomarker for poor prognosis in patients with lung cancer.
\end{abstract}

\section{Introduction}

Lung cancer is a leading cause of morbidity and mortality worldwide $(1,2)$. The two main types of lung cancer are non-small-cell lung cancer (NSCLC) and small-cell lung cancer (SCLC), which account for 85 and $15 \%$ of all cases, respectively (3). However, despite improvements in the

Correspondence to: Professor Jiuwei Cui, Cancer Center, The First Hospital of Jilin University, No. 71 Xinmin Street, Changchun, Jilin 130021, P.R. China

E-mail: cuijw@jlu.edu.cn

Key words: neutrophil-to-lymphocyte ratio, inflammation, lung cancer, prognosis, meta-analysis modalities for diagnosing and treating lung cancer, the prognosis remains poor. Thus, novel and effective prognostic factors, which may allow clinicians to use effective therapeutic strategies, are urgently needed.

The traditional prognostic markers for lung cancer prognosis include patient age (4), sex (5), smoking (6), and TNM classification (7). There are also novel biomarkers that are able to predict prognosis and guide clinical treatment, including elevated levels of carcinoembryonic antigen, cytokeratin-19 fragments, squamous cell carcinoma antigen, progastrin-releasing peptide, tumor M2-pyruvate kinase, and C-reactive protein (8). However, patients with the same TNM stage may have a different prognosis (9), whereas some of the abovementioned prognostic biomarkers are costly and, thus, not included in routine tests for the majority of the patients.

During recent years, an increasing number of studies have revealed an association between systemic inflammation and tumorigenesis-related factors, including tumor angiogenesis, progression, invasion and metastasis (10-13). Accumulating evidence also indicates that tumor-associated inflammation may be detected in the peripheral blood as neutrophilia and/or lymphopenia (14). This finding suggests that the levels of neutrophils and lymphocytes may function as a combined factor, which may more accurately reflect the inflammatory response, compared with a single factor. Thus, the neutrophil-to-lymphocyte ratio (NLR) has been developed as a novel indicator of inflammation, and an elevated NLR may be associated with a poor prognosis (15). Moreover, routine laboratory and blood tests are performed during the pre-treatment work-up for all patients, and these results may be used to evaluate the patient's NLR. Therefore, NLR is a minimally invasive and inexpensive biomarker that may be used to predict prognosis among patients with lung cancer. However, there is controversy regarding whether NLR is a convincing or effective clinical indicator, and the association between NLR and lung cancer remains unclear. The aim of the present meta-analysis was to evaluate whether NLR is of value for predicting the prognosis of lung cancer.

\section{Data collection methods}

Search strategy. A systematic search was performed through the PubMed, Embase and Cochrane Library databases from inception up to July 30, 2016. The search used the following terms: Neutrophil-to-lymphocyte ratio, neutrophil to lymphocyte ratio, 
neutrophil-lymphocyte ratio, neutrophil lymphocyte ratio, and $\mathrm{MeSH}$ terms (lung neoplasms AND prognosis). The reference lists from the identified reports were also reviewed, in order to retrieve other potentially relevant studies.

Study selection criteria. Articles were considered eligible if they fulfilled the following criteria: i) The patients were pathologically diagnosed with lung cancer (NSCLC or SCLC); ii) the study investigated the association between pretreatment NLR and various outcomes, including overall survival (OS), progression-free survival (PFS), disease-free survival, or recurrence-free survival; iii) reported hazard ratios (HRs) and 95\% confidence intervals (CIs) or provided sufficient information to estimate the HRs and 95\% CIs; and iv) the full text was accessible and written in English.

Data extraction. All the retrieved articles were independently reviewed by two investigators ( $\mathrm{Yu}$ Yu and Lei Qian). The extracted data included the first author's name, year of publication, study duration, country, ethnicity, sample size, sex, age, stage, tumor type, follow-up period, treatment, study design, and cut-off value for elevated NLR with the HRs and/or 95\% CIs. Disagreements were discussed and resolved through consensus.

Statistical analysis. Based on the methods of Tierney et al (16), the HRs and $95 \%$ CIs were estimated or extracted to evaluate the significance of NLR according to OS and PFS. A poorer prognosis was defined as an elevated NLR being associated with an HR of $>1$. Heterogeneity of the pooled results was tested using Cochran's Q test and Higgins' I-squared statistic, with an $\mathrm{I}^{2}$ of $>50 \%$ representing significant heterogeneity. The pooled HRs and 95\% CIs were calculated using a random-effects model (Der Simonian-Laird method) or a fixed-effects model (Mantel-Haenszel method), as appropriate The random-effects model was defined as the preferred method when heterogeneity was detected. Inter-study heterogeneity was also investigated using subgroup analysis and meta-regression analysis. Sensitivity analysis was also performed to evaluate the stability and credibility of the results. Publication bias was assessed using Egger's funnel plot. All statistical tests were two-sided and P-values $<0.05$ were considered to indicate statistically significant differences. All statistical analyses were performed using Stata software, version 13.1 (StataCorp LP, College Station, TX, USA).

\section{Results}

Study characteristics. The study selection flow chart is shown in Fig. 1. The initial search through the PubMed, Embase and Cochrane Library databases identified 125 studies. After excluding duplicate reports, irrelevant reports, reviews and conference abstracts, aa total of 37 full-text reports were included in the evaluation. Subsequently, 19 studies were excluded for the following reasons: 7 studies failed to provide sufficient data for the analyses, 6 studies did not report the NLR cut-off value, 4 studies did not report specific NLR data according to OS, and 2 studies had data duplication. Thus, the final analyses included 18 studies (19 cohorts) (17-34) with 7,219 patients, published between 2009-2016. The patients in the report by Botta et al (22) were split into two independent cohorts (Botta 1 and Botta 2) due to the cohort design of the article.

The characteristics of the 18 studies are summarized in Table I. A total of 7 studies were conducted in Western countries, including the UK (17,26,34), USA (28), Spain (20) and Italy $(22,34), 1$ study was performed in Turkey (23), and 10 studies were performed in East Asian countries, including Japan $(18,19,31,32)$, Korea $(21,25)$ and China $(24,27,30,33)$. The study published by Mitchell et al (29) included patient data from 33 countries. The included studies evaluated 16 populations of patients with NSCLC (17-24,26-29,31-34) and 2 populations of patients with $\operatorname{SCLC}(25,30)$. The 2 studies on SCLC reported tumor staging information as limited and extensive disease, so only the staging information from the studies regarding NSCLC were considered Only 1 study by Sarraf et al (17) evaluated all tumor stages, 8 studies evaluated early-stage tumors (23,26-29,31-33), and 6 studies evaluated late-stage tumors $(18,20-22,24,34)$. The study by Tomita et al (19) evaluated stages IA/III/IV. The cut-off values for elevated NLR ranged from 2.5 to 5 .

NLR and $O S$ in lung cancer. A total of 17 studies with 17 cohorts (17-21,23-34) including 7,107 patients evaluated the association between elevated pretreatment NLR and OS among patients with lung cancer. The random-effects model was used for this analysis, as significant heterogeneity was detected $\left(\mathrm{I}^{2}=84.2 \%, \mathrm{P}_{\text {heterogeneity }}<0.001\right)$. The pooled HR was 1.46 (95\% CI: 1.30-1.64, P<0.001; Fig. 2), which suggested that elevated pretreatment NLR predicted poor OS after treatment for lung cancer.

NLR and PFS in lung cancer. A total of 7 studies with 8 cohorts $(18,21,22,24,25,30,34)$ including 1,581 patients evaluated the association between elevated pretreatment NLR and PFS among patients with lung cancer. Significant heterogeneity was also detected among these studies $\left(\mathrm{I}^{2}=76.2 \%\right.$, $\left.\mathrm{P}_{\text {heterogeneity }}<0.001\right)$. A pooled HR of 1.42 (95\% CI: 1.15-1.75, $\mathrm{P}=0.001$; Fig. 3) suggested that elevated pretreatment NLR predicted shorter PFS after treatment for lung cancer.

Subgroup analysis. A subgroup analysis was performed to identify the possible reason(s) for the significant heterogeneity in the meta-analysis (Table II). The OS-related subgroup analysis included 7 subgroups: Treatment (surgery and non-surgery), ethnicity (Caucasian and Asian), tumor stage (late stage: IIIB-IV; and early stage: I-IIIA), sample size $(<200$ and $\geq 200)$, NLR cut-off value $(<4$ and $\geq 4)$, tumor type (NSCLC and SCLC), and analysis method (multivariate and univariate). The results consistently demonstrated that elevated pretreatment NLR predicted poor OS after treatment for lung cancer. The PFS-related subgroup analysis included 5 subgroups (ethnicity, sample size, cut-off value, tumor type and analysis method), and the combined results were similar to those for OS. Interestingly, an NLR cut-off of $\geq 4$ was found to be associated with significantly lower heterogeneity $\left(\mathrm{OS}: \mathrm{I}^{2}=14.3 \%, \mathrm{P}_{\text {heterogeneity }}=0.323\right.$; PFS: $\left.\mathrm{I}^{2}=57.2 \%, \mathrm{P}_{\text {heterogeneity }}=0.029\right)$, suggesting that an NLR of $\geq 4$ was a useful prognostic indicator for both OS and PFS.

Heterogeneity. Meta-regression analysis was performed to explore the potential source(s) of heterogeneity in the 


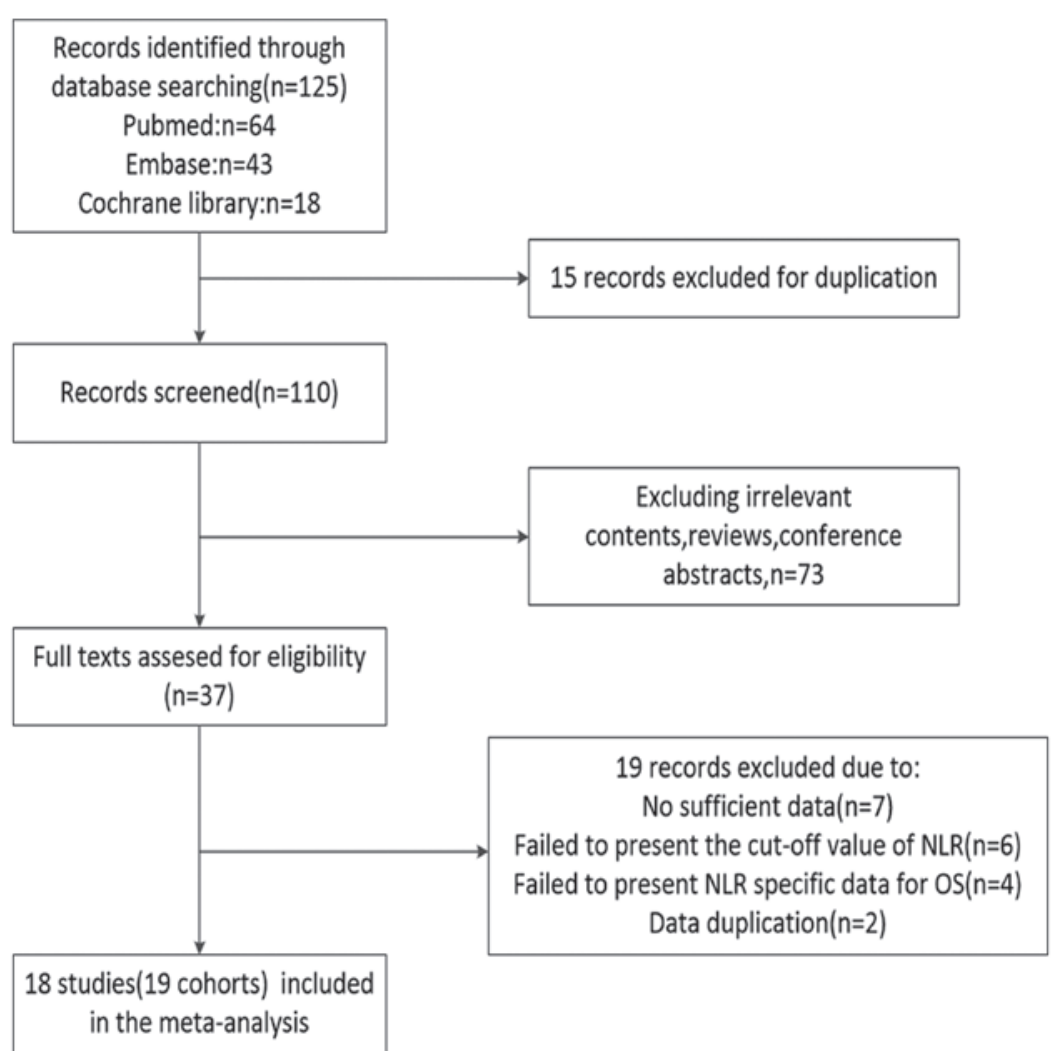

Figure 1. Flow chart of the included studies. NLR, neutrophil-to-lymphocyte ratio; OS, overall survival.

associations of NLR with OS and PFS. The results revealed that heterogeneity in the OS results was not significantly affected by treatment $(\mathrm{P}=0.725)$, ethnicity $(\mathrm{P}=0.976)$, tumor stage $(\mathrm{P}=0.305)$, sample size $(\mathrm{P}=0.156)$, cut-off value $(\mathrm{P}=0.807)$, or tumor type $(\mathrm{P}=0.884)$. The results also revealed that heterogeneity in the PFS results was not significantly affected by ethnicity $(\mathrm{P}=0.696)$, sample size $(\mathrm{P}=0.942)$, cut-off value $(\mathrm{P}=0.137)$, or tumor type $(\mathrm{P}=0.844)$.

Publication bias and sensitivity analysis. Egger's test revealed statistically significant publication bias for both OS and PFS (both $\mathrm{P}<0.05$ ). A sensitivity analysis was performed by sequentially removing each study, and evaluating whether any individual study significantly affected the results. However, the pooled HRs and $95 \%$ CIs revealed that no single study significantly affected OS or PFS (Figs. 4 and 5).

\section{Discussion}

The present meta-analysis evaluated the prognostic value of elevated pretreatment NLR in 18 studies and 7,219 patients with lung cancer. To the best of our knowledge, this is the largest meta-analysis regarding this topic to date. The pooled HRs suggest that elevated pretreatment NLR was associated with poor $\mathrm{OS}(\mathrm{HR}=1.46,95 \% \mathrm{CI}: 1.30-1.64)$ and poor $\mathrm{PFS}$ (HR=1.42, 95\% CI: 1.15-1.75). Furthermore, the subgroup analysis revealed that an elevated pretreatment NLR of $\geq 4$ effectively predicted poor OS and PFS after treatment for lung cancer, regardless of the analytical method. One previous study had used an NLR cut-off of 4 (35), and another study used a cut-off of 5 (35). Our results confirmed that an NLR of 4 is a more stable threshold for predicting prognosis, as our subgroup analysis with an NLR of $\geq 4$ revealed significantly lower heterogeneity. We suggest that the setting of the reliable threshold of the NLR for predicting prognosis of lung cancer may be very helpful for clinical use. Of note, NLR may also better predict a poor OS for patients who undergo surgery $(\mathrm{HR}=1.50,95 \%$ CI: $1.21-1.84)$ or patients with early-stage tumors ( $\mathrm{HR}=1.64$, 95\% CI: 1.37-1.97), suggesting that NLR may be used as an independent prognostic indicator to monitor the postoperative outcome of patients with early-stage lung cancer. In cases with SCLC, NLR provided significantly improved prognostic value, without any heterogeneity in the OS and PFS analysis. The use of NLR may be promising in evaluating the prognosis of SCLC patients. However, additional studies are required to validate this association, as only 2 studies evaluating SCLC cases were identified.

Accumulating evidence suggests that a dysregulated inflammatory response plays a vital role in cancer (36). Infiltration by immune cells is increasingly accepted as an important part of the tumor microenvironment, which may lead to cancer-related inflammation (37). In this context, NLR has recently been introduced as a simple index of the systemic inflammatory response, as inflammation leads to more neutrophils and fewer lymphocytes in the peripheral blood. Together, these changes result in an elevated NLR. Neutrophils are the dominant leukocytes in the blood, and are the first line of defense against inflammation and infection (38). Neutrophil infiltration is also observed in a number of tumor types, and tumor-associated neutrophils in lung cancer are associated with malignant potential and a poor prognosis (39). According to Proctor et al, NLR may be a more sensitive 


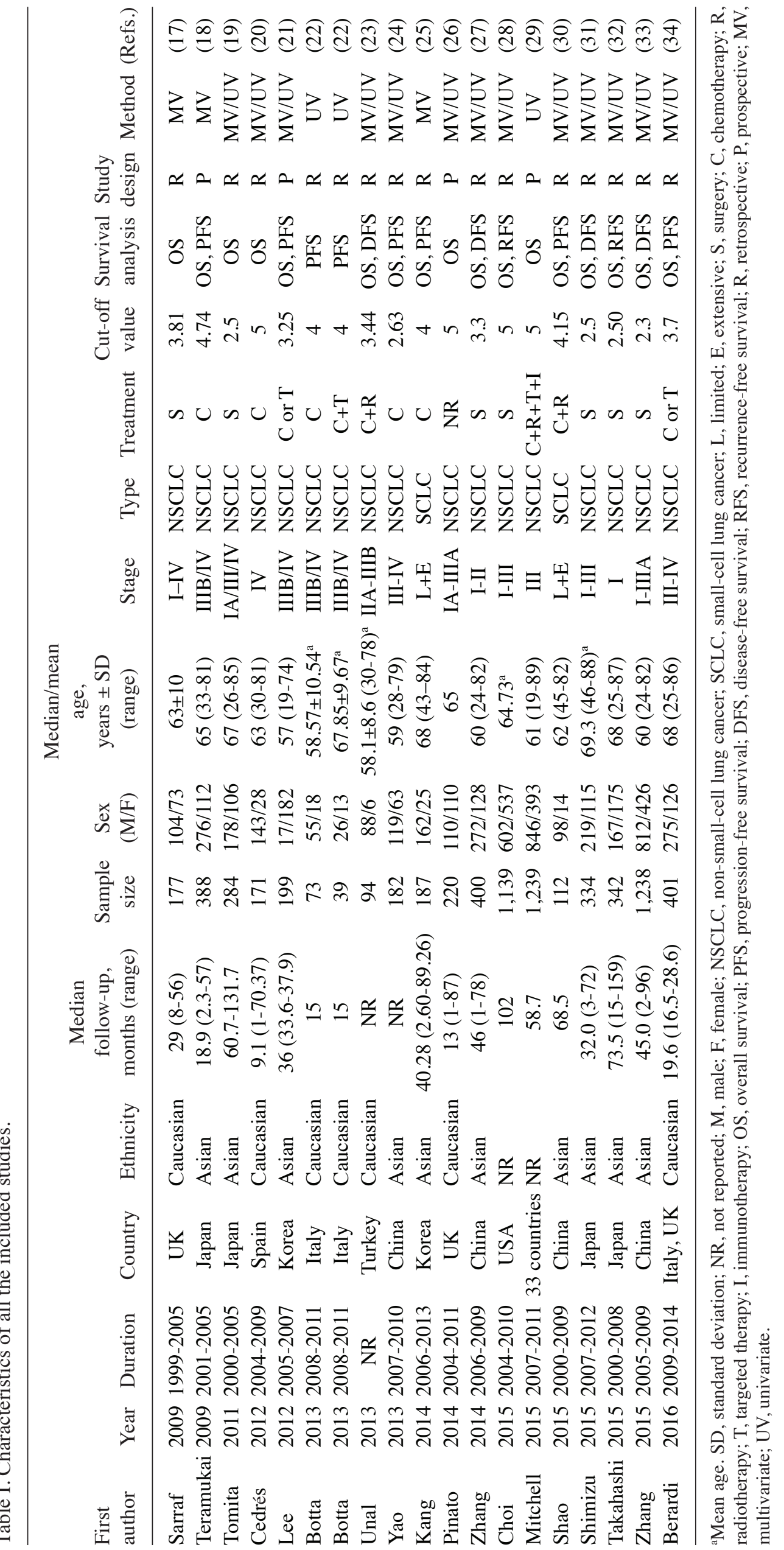




\begin{tabular}{|c|c|c|c|}
\hline \multicolumn{2}{|l|}{ Study } & \multicolumn{2}{|r|}{$\%$} \\
\hline \multicolumn{2}{|l|}{ ID } & \multirow{2}{*}{$\begin{array}{l}\mathrm{HR}(95 \% \mathrm{Cl}) \\
1.10(1.03,1.17)\end{array}$} & \multirow{2}{*}{$\begin{array}{l}\text { Weight } \\
9.76\end{array}$} \\
\hline Sarraf (2009) & $\leftarrow$ & & \\
\hline Teramukai (2009) & $\longrightarrow$ & $1.56(1.09,2.24)$ & 5.10 \\
\hline Tomita (2011) & $\rightarrow$ & $1.29(1.05,1.57)$ & 7.72 \\
\hline Cedres (2012) & $\rightarrow$ & $1.40(1.10,2.10)$ & 5.64 \\
\hline Lee (2012) & $\bullet$ & $1.05(1.00,1.10)$ & 9.89 \\
\hline Unal (2013) & & $1.81(1.16,2.82)$ & 4.06 \\
\hline Yao (2013) & & $1.76(1.10,2.83)$ & 3.74 \\
\hline Kang (2014) & & $1.47(1.01,2.12)$ & 4.97 \\
\hline Pinato (2014) & & $3.80(1.60,8.90)$ & 1.54 \\
\hline Zhang (2014) & $\rightarrow$ & $2.08(1.32,3.27)$ & 3.94 \\
\hline Choi (2015) & & $1.69(1.27,2.23)$ & 6.31 \\
\hline Mitchell (2015) & + & $1.12(0.94,1.34)$ & 8.14 \\
\hline Shao (2015) & $\longrightarrow$ & $1.56(1.16,1.96)$ & 6.62 \\
\hline Shimizu (2015) & $\rightarrow$ & $1.60(1.04,2.54)$ & 4.03 \\
\hline Takahashi (2015) & & $2.14(1.31,3.52)$ & 3.55 \\
\hline Zhang (2015) & $\leftarrow$ & $1.53(1.46,1.78)$ & 9.34 \\
\hline Berardi (2016) & $\frac{1}{10}$ & $1.74(1.26,2.41)$ & 5.63 \\
\hline Overall $(1-$ squared $=84.2 \%, p=0.000)$ & $\diamond$ & $1.46(1.30,1.64)$ & 100.00 \\
\hline \multicolumn{4}{|c|}{ NOTE: Weights are from random effects analysi\$ } \\
\hline 0.112 & 1 & & \\
\hline
\end{tabular}

Figure 2. Forest plot of the association between elevated pretreatment neutrophil-to-lymphocyte ratio and overall survival among patients with lung cancer. HR, hazard ratio; $\mathrm{CI}$, confidence interval.

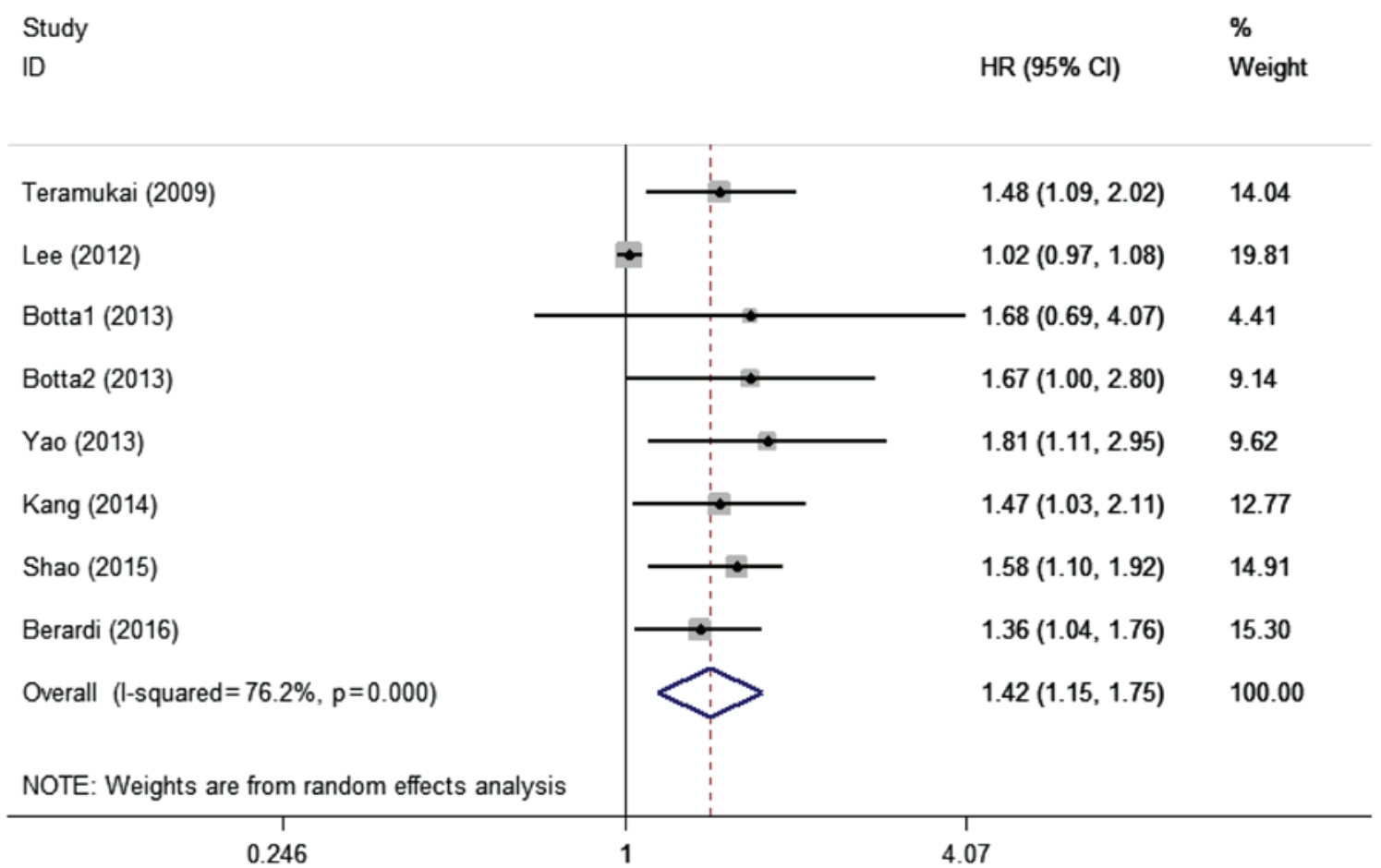

Figure 3. Forest plot of the association between elevated pretreatment neutrophil-to-lymphocyte ratio and progression-free survival among patients with lung cancer. HR, hazard ratio; CI, confidence interval.

composite index, compared with white blood cell count (40). By contrast, elevated levels of tumor-infiltrating lymphocytes are considered to be associated with a better prognosis (41), and decreasing levels of tumor-infiltrating lymphocytes are associated with a poor prognosis in lung cancer (42). Thus, the balance between the conflicting inflammatory responses in tumors is likely an effective predictor of prognosis (43), and NLR appears to be a superior index of the balance between the inflammatory response and tumor immune status, compared with individual neutrophil or lymphocyte counts.

The present study revealed significant heterogeneity in the available data, which was not explained by the subgroup 
Table II. Summary of the meta-analysis results.

A, Overall survival

\begin{tabular}{|c|c|c|c|c|c|c|c|}
\hline \multirow[b]{2}{*}{ Subgroup } & \multirow[b]{2}{*}{ Number } & \multicolumn{2}{|c|}{ Random-effects model } & \multicolumn{2}{|c|}{ Fixed-effects model } & \multicolumn{2}{|c|}{ Heterogeneity } \\
\hline & & HR $(95 \%$ CI $)$ & P-value & HR $(95 \%$ CI $)$ & P-value & $\mathrm{I}^{2}(\%)$ & $\mathrm{P}_{\mathrm{h}}$ \\
\hline \multicolumn{8}{|l|}{ Treatment } \\
\hline Surgery & 7 & $1.57(1.21-1.95)$ & $<0.001$ & $1.25(1.19-1.31)$ & $<0.001$ & 88.3 & $<0.001$ \\
\hline Non-surgery & 10 & $1.42(1.21-1.68)$ & $<0.001$ & $1.11(1.06-1.16)$ & $<0.001$ & 75.9 & $<0.001$ \\
\hline \multicolumn{8}{|l|}{ Ethnicity } \\
\hline Caucasian & 5 & $1.58(1.15-2.17)$ & 0.005 & $1.14(1.08-1.22)$ & $<0.001$ & 80.9 & $<0.001$ \\
\hline Asian & 10 & $1.51(1.26-1.81)$ & $<0.001$ & $1.17(1.12-1.21)$ & $<0.001$ & 87.7 & $<0.001$ \\
\hline \multicolumn{8}{|l|}{ Tumor stage } \\
\hline Late & 5 & $1.43(1.09-1.87)$ & 0.1 & $1.08(1.03-1.13)$ & 0.001 & 80.2 & $<0.001$ \\
\hline Early & 8 & $1.64(1.37-1.97)$ & $<0.001$ & $1.50(1.39-1.62)$ & $<0.001$ & 65.9 & 0.005 \\
\hline \multicolumn{8}{|l|}{ Sample size, $n$} \\
\hline$<200$ & 7 & $1.25(1.11-1.41)$ & $<0.001$ & $1.09(1.05-1.13)$ & $<0.001$ & 74.1 & 0.001 \\
\hline$\geq 200$ & 10 & $1.56(1.35-1.79)$ & $<0.001$ & $1.48(1.38-1.59)$ & $<0.001$ & 60.3 & 0.007 \\
\hline \multicolumn{8}{|l|}{ Cut-off value } \\
\hline$<4$ & 10 & $1.44(1.24-1.66)$ & $<0.001$ & $1.14(1.10-1.18)$ & $<0.001$ & 88.2 & $<0.001$ \\
\hline$\geq 4$ & 5 & $1.56(1.31-1.85)$ & $<0.001$ & $1.55(1.32-1.81)$ & $<0.001$ & 14.3 & 0.323 \\
\hline \multicolumn{8}{|l|}{ Type } \\
\hline NSCLC & 15 & $1.45(1.28-1.64)$ & $<0.001$ & $1.16(1.12-1.20)$ & $<0.001$ & 85.2 & $<0.001$ \\
\hline SCLC & 2 & $1.53(1.23-1.89)$ & $<0.001$ & $1.53(1.23-1.89)$ & $<0.001$ & 0 & 0.792 \\
\hline \multicolumn{8}{|l|}{ Method } \\
\hline Multivariate & 16 & $1.50(1.32-1.70)$ & $<0.001$ & $1.17(1.13-1.21)$ & $<0.001$ & 85.2 & $<0.001$ \\
\hline Univariate & 12 & $1.51(1.28-1.78)$ & $<0.001$ & $1.17(1.13-1.22)$ & $<0.001$ & 87.4 & $<0.001$ \\
\hline
\end{tabular}

B, Progression-free survival

\begin{tabular}{|c|c|c|c|c|c|c|c|}
\hline \multicolumn{8}{|l|}{ Ethnicity } \\
\hline Caucasian & 3 & $1.43(1.14-1.80)$ & 0.002 & $1.43(1.14-1.80)$ & 0.002 & 0 & 0.735 \\
\hline Asian & 5 & $1.40(1.07-1.83)$ & 0.015 & $1.06(1.01-1.12)$ & 0.024 & 82.1 & $<0.001$ \\
\hline \multicolumn{8}{|l|}{ Sample size, $\mathrm{n}$} \\
\hline$<200$ & 6 & $1.44(1.09-1.90)$ & 0.1 & $1.06(1.00-1.11)$ & 0.034 & 77.1 & 0.001 \\
\hline$\geq 200$ & 2 & $1.41(1.15-1.72)$ & 0.001 & $1.41(1.15-1.72)$ & 0.001 & 0 & 0.683 \\
\hline \multicolumn{8}{|l|}{ Cut-off value } \\
\hline$<4$ & 3 & $1.27(0.93-1.72)$ & 0.128 & $1.04(0.99-1.09)$ & 0.158 & 88.2 & $<0.001$ \\
\hline$\geq 4$ & 5 & $1.54(1.13-1.82)$ & $<0.001$ & $1.54(1.30-1.82)$ & $<0.001$ & 57.2 & 0.029 \\
\hline \multicolumn{8}{|l|}{ Type } \\
\hline NSCLC & 6 & $1.38(1.08-1.75)$ & 0.1 & $1.06(1.00-1.11)$ & 0.4 & 72.9 & 0.002 \\
\hline SCLC & 2 & $1.54(1.24-1.92)$ & $<0.001$ & $1.54(1.24-1.92)$ & $<0.001$ & 0 & 0.761 \\
\hline \multicolumn{8}{|l|}{ Method } \\
\hline Multivariate & 6 & $1.38(1.10-1.74)$ & 0.027 & $1.07(1.02-1.13)$ & 0.008 & 80.5 & $<0.001$ \\
\hline Univariate & 5 & $1.35(1.03-1.75)$ & 0.002 & $1.05(1.00-1.11)$ & 0.046 & 77 & 0.002 \\
\hline
\end{tabular}

OS, overall survival; PFS, progression-free survival; NSCLC, non-small-cell lung cancer; SCLC, small-cell lung cancer; HR, hazard ratio; CI, confidence interval; $\mathrm{P}_{\mathrm{h}}$, P-value of Q-test for heterogeneity.

analysis. Thus, we hypothesized that the heterogeneity may be associated with confounding or unconsidered factors. In this context, a number of traditional factors may be of value for predicting prognosis in lung cancer cases, and some of these factors may exert synergistic effects, particularly factors that are associated with host status. However, the studies in the 


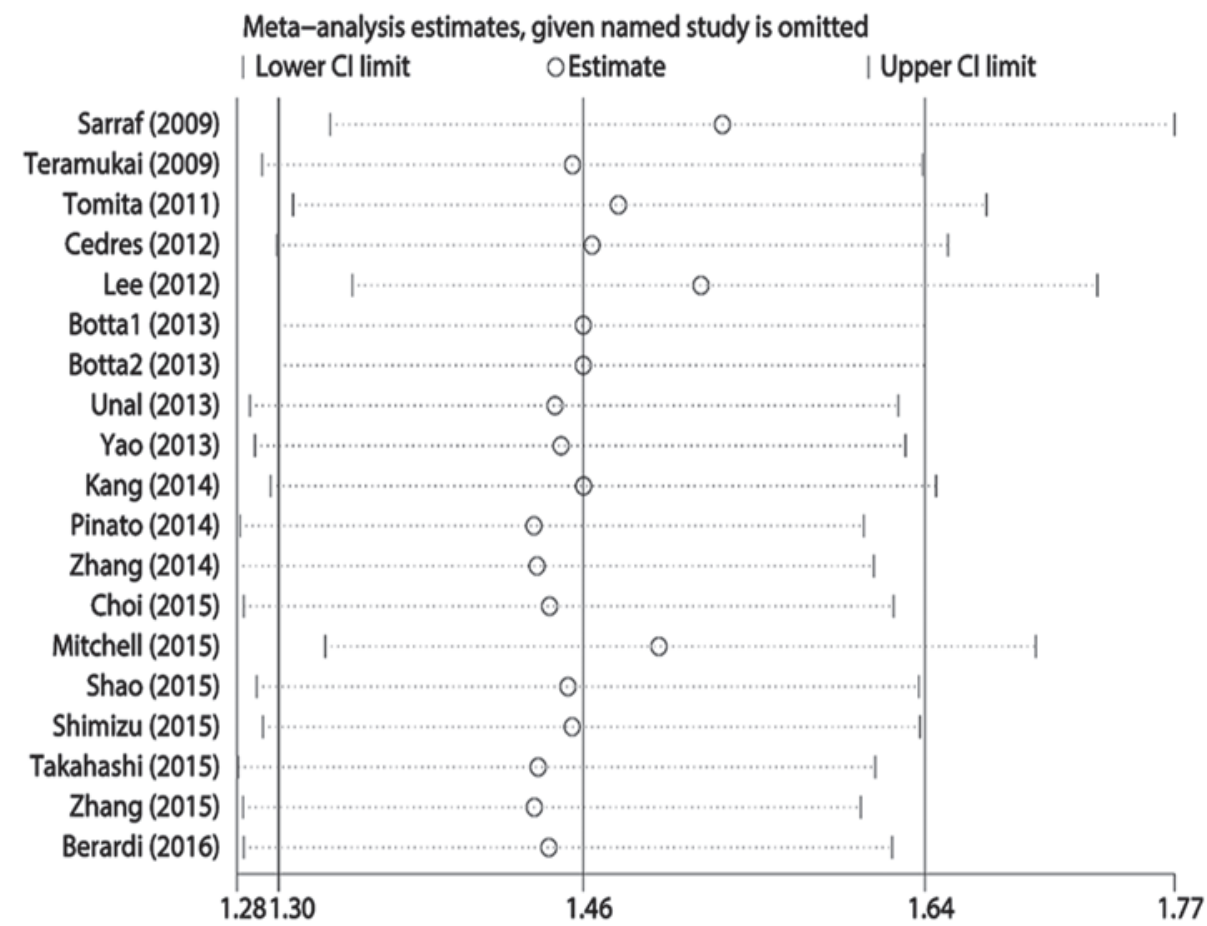

Figure 4. Sensitivity analysis of the included studies for the association of neutrophil-to-lymphocyte ratio and overall survival. CI, confidence interval.

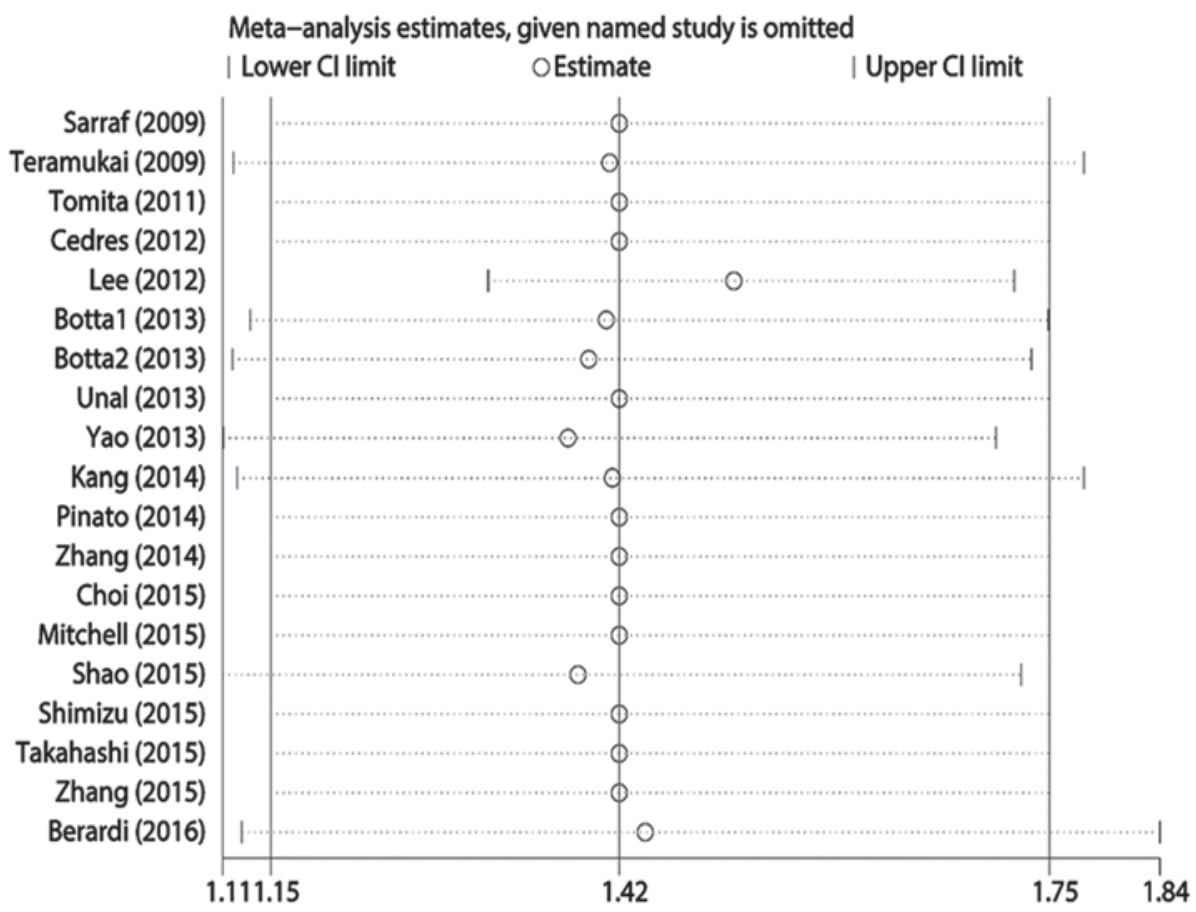

Figure 5. Sensitivity analysis of the included studies for the association of neutrophil-to-lymphocyte ratio and progression-free survival. CI, confidence interval.

present meta-analysis did not take into consideration factors in combination with NLR, and there were insufficient data to perform additional subgroup analysis. Thus, we considered other studies' results to explore these potential factors and their prognostic value in lung cancer. For example, young Japanese patients (aged $\leq 50$ years) exhibit better survival after surgery for lung cancer, compared with older patients, which may be associated with the significantly better performance status among younger patients (44). In addition, a study of two cohorts in Australia and America revealed that male sex was independently associated with poor prognosis in NSCLC (5). Furthermore, another meta-analysis suggested that smoking cessation improves prognosis for patients with early-stage lung cancer, and this result may also be associated with a poor prognosis among male patients, as they are relatively heavy smokers (45). Moreover, smoking may promote the progression 
of both early- and late-stage lung cancer through DNA alterations and modified protein expression (6). According to Kanarek et al (46), patients may also achieve a rapid reduction in tumor burden if they have a short referral interval and pre-surgery delay, which may be associated with continued smoking. Thus, all these factors may be useful in predicting the prognosis of lung cancer, with the exception of histological classification after radiotherapy (47) or chemotherapy (48). In addition, there is a clear inverse correlation between NLR and nutritional status (31), which indicates that NLR in the inflammatory response may be a host-related prognostic factor. Therefore, as NLR may be affected by various factors and their combinations, large-scale studies are required to elucidate the mechanism(s) underlying the association between elevated NLR and prognosis after treatment for lung cancer.

Several recent meta-analyses have used NLR as an important prognostic factor for various cancer types with different but similar cut-off values, including esophageal cancer (2-5) (49), breast cancer (3) (50), gastric cancer (3) (51), hepatocellular carcinoma (3-4) (52), pancreatic cancer (2.3-5) (53), colorectal cancer (5) (54), renal cell carcinoma (3) (55) and prostate cancer (3) (56). All these studies have reported that NLR may be a promising prognostic factor for that specific cancer. Moreover, NLR may be associated with diseases other than cancer, such as diabetes mellitus and cardiovascular disease (57). Thus, NLR is likely of value for predicting prognosis in a wide range of inflammation-associated diseases.

There are certain limitations regarding the present meta-analysis that should be addressed. First, only 4 prospective studies were identified, whereas 13 studies (14 cohorts) used a retrospective design, which increases the risk of bias. Second, substantial heterogeneity was observed in the various studies, which was associated with various confounding factors, such as ethnicity, sex, treatment method, follow-up period, age distribution, and NLR cut-off value. However, this significant heterogeneity was not attributable to a single factor in the subgroup analysis, meta-regression, and sensitivity analysis, suggesting that the heterogeneity may be associated with the inter-related factors that were discussed in the previous paragraphs. Third, only 2 studies on SCLC were identified, and included a limited amount of data for the PFS-related analysis, which increases the risk of bias in our findings. Fourth, the association between the NLR and clinicopathological parameters (e.g., lymph node metastasis) or pathological patterns was not analyzed. Fifth, only 3 studies evaluated NLR using multivariate analysis, and the remaining studies either performed univariate analysis alone or a combination of multivariate and univariate analysis. Sixth, significant publication bias was identified, which was likely associated with the language restriction and the increased likelihood that reports with positive results would be published.

In conclusion, the present meta-analysis demonstrated that elevated pretreatment NLR was associated with prognosis among patients with lung cancer. Thus, NLR may be an easily accessible and effective prognostic biomarker in lung cancer, as it may be evaluated during routine blood tests. However, the specific mechanism underlying its prognostic value remains unclear, as significant heterogeneity was observed in the present meta-analysis. Therefore, additional well-designed large-scale studies are required to clearly determine the prognostic role of NLR in lung cancer.

\section{Acknowledgements}

The present study was supported by the Jilin Provincial Science and Technology Department (grant no. 20150101176 to J.C.), the National Health and Family Planning Commission of the People's Republic of China (grant no. ZX-07-C2016004), and the National Key Research and Development Program of China (grant no. 2016YFC1303800).

\section{References}

1. Siegel RL, Miller KD and Jemal A: Cancer statistics, 2016. CA Cancer J Clin 66: 7-30, 2016.

2. Torre LA, Bray F, Siegel RL, Ferlay J, Lortet-Tieulent J and Jemal A: Global cancer statistics, 2012. CA Cancer J Clin 65: 87-108, 2015

3. Dela Cruz CS, Tanoue LT and Matthay RA: Lung cancer: Epidemiology, etiology, and prevention. Clin Chest Med 32: 605-644, 2011.

4. Tas F, Ciftci R, Kilic L and Karabulut S: Age is a prognostic factor affecting survival in lung cancer patients. Oncol Lett 6: 1507-1513, 2013.

5. Wainer Z, Wright GM, Gough K, Daniels MG, Choong P, Conron M, Russell PA, Alam NZ, Ball D and Solomon B: Impact of sex on prognostic host factors in surgical patients with lung cancer. ANZ J Surg: doi: 10.1111/ans.13728.

6. Yoshino I and Maehara Y: Impact of smoking status on the biological behavior of lung cancer. Surg Today 37: 725-734, 2007.

7. Sobin LH, Gospodarowicz MK and Wittekind C: TNM classification of malignant tumours. 7th edition. Wiley-Blackwell, 2009.

8. Greenberg AK and Lee MS: Biomarkers for lung cancer: Clinical uses. Curr Opin Pulm Med 13: 249-255, 2007.

9. Yoon HM, Ryu KW, Nam BH, Cho SJ, Park SR, Lee JY, Lee JH, Kook MC, Choi IJ and Kim YW: Is the new seventh AJCC/UICC staging system appropriate for patients with gastric cancer? J Am Co Surg 214: 88-96, 2012

10. Balkwill $\mathrm{F}$ and Mantovani A: Inflammation and cancer: Back to Virchow? Lancet 357: 539-545, 2001.

11. Elinav E, Nowarski R, Thaiss CA, Hu B, Jin C and Flavell RA: Inflammation-induced cancer: Crosstalk between tumours, immune cells and microorganisms. Nat Rev Cancer 13: 759-771, 2013.

12. Coussens LM and Werb Z: Inflammation and cancer. Nature 420: 860-867, 2002.

13. Grivennikov SI, Greten FR and Karin M: Immunity, inflammation, and cancer. Cell 140: 883-899, 2010.

14. Kumar R, Geuna E, Michalarea V, Guardascione M, Naumann U, Lorente D, Kaye SB and de Bono JS: The neutrophil-lymphocyte ratio and its utilisation for the management of cancer patients in early clinical trials. Br J Cancer 112: 1157-1165, 2015.

15. Guthrie GJ, Charles KA, Roxburgh CS, Horgan PG, McMillan DC and Clarke SJ: The systemic inflammation-based neutrophil-lymphocyte ratio: Experience in patients with cancer. Crit Rev Oncol Hematol 88: 218-230, 2013.

16. Tierney JF, Stewart LA, Ghersi D, Burdett S and Sydes MR: Practical methods for incorporating summary time-to-event data into meta-analysis. Trials 8: 16, 2007.

17. Sarraf KM, Belcher E, Raevsky E, Nicholson AG, Goldstraw P and Lim E: Neutrophil/lymphocyte ratio and its association with survival after complete resection in non-small cell lung cancer. J Thorac Cardiovasc Surg 137: 425-428, 2009.

18. Teramukai S, Kitano T, Kishida Y, Kawahara M, Kubota K, Komuta K, Minato K, Mio T, Fujita Y, Yonei T, et al: Pretreatment neutrophil count as an independent prognostic factor in advanced non-small-cell lung cancer: An analysis of Japan Multinational Trial Organisation LC00-03. Eur J Cancer 45: 1950-1958, 2009.

19. Tomita M, Shimizu T, Ayabe T, Yonei A and Onitsuka T: Preoperative neutrophil to lymphocyte ratio as a prognostic predictor after curative resection for non-small cell lung cancer. Anticancer Res 31: 2995-2998, 2011. 
20. Cedrés S, Torrejon D, Martinez A, Martinez P, Navarro A, Zamora E, Mulet-Margalef N and Felip E: Neutrophil to lymphocyte ratio (NLR) as an indicator of poor prognosis in stage IV non-small cell lung cancer. Clin Transl Oncol 14: 864-869, 2012.

21. Lee Y, Kim SH, Han JY, Kim HT, Yun T and Lee JS: Early neutrophil-to-lymphocyte ratio reduction as a surrogate marker of prognosis in never smokers with advanced lung adenocarcinoma receiving gefitinib or standard chemotherapy as first-line therapy. J Cancer Res Clin Oncol 138: 2009-2016, 2012.

22. Botta C, Barbieri V, Ciliberto D, Rossi A, Rocco D, Addeo R, Staropoli N, Pastina P, Marvaso G, Martellucci I, et al: Systemic inflammatory status at baseline predicts bevacizumab benefit in advanced non-small cell lung cancer patients. Cancer Bio Ther 14: 469-475, 2013

23. Unal D, Eroglu C, Kurtul N, Oguz A and Tasdemir A: Are neutrophil/lymphocyte and platelet/lymphocyte rates in patients with non-small cell lung cancer associated with treatment response and prognosis? Asian Pac J Cancer Prev 14: 5237-5242, 2013

24. Yao Y, Yuan D, Liu H, Gu X and Song Y: Pretreatment neutrophil to lymphocyte ratio is associated with response to therapy and prognosis of advanced non-small cell lung cancer patients treated with first-line platinum-based chemotherapy. Cancer Immunol Immunother 62: 471-479, 2013.

25. Kang MH, Go SI, Song HN, Lee A, Kim SH, Kang JH, Jeong BK, Kang KM, Ling $H$ and Lee GW: The prognostic impact of the neutrophil-to-lymphocyte ratio in patients with small-cell lung cancer. Br J Cancer 111: 452-460, 2014.

26. Pinato DJ, Shiner RJ, Seck1 MJ, Stebbing J, Sharma R and Mauri FA: Prognostic performance of inflammation-based prognostic indices in primary operable non-small cell lung cancer. $\mathrm{Br}$ J Cancer 110: 1930-1935, 2014.

27. Zhang T, Jiang Y, Qu X, Shen H, Liu Q and Du J: Evaluation of preoperative hematologic markers as prognostic factors and establishment of novel risk stratification in resected $\mathrm{pNO}$ non-small-cell lung cancer. PLoS One 9: e111494, 2014

28. Choi JE, Villarreal J, Lasala J, Gottumukkala V, Mehran RJ, Rice D, Yu J, Feng L and Cata JP: Perioperative neutrophil: Lymphocyte ratio and postoperative NSAID use as predictors of survival after lung cancer surgery: A retrospective study. Cancer Med 4: 825-833, 2015

29. Mitchell PL, Thatcher N, Socinski MA, Wasilewska-Tesluk E, Horwood K, Szczesna A, Martín C, Ragulin Y, Zukin M, Helwig C, et al: Tecemotide in unresectable stage III non-small-cell lung cancer in the phase III START study: Updated overall survival and biomarker analyses. Ann Oncol 26 $1134-1142,2015$

30. Shao N and Cai Q: High pretreatment neutrophil-lymphocyte ratio predicts recurrence and poor prognosis for combined small cell lung cancer. Clin Transl Oncol 17: 772-778, 2015.

31. Shimizu K, Okita R, Saisho S, Maeda A, Nojima Y and Nakata M: Preoperative neutrophil/lymphocyte ratio and prognostic nutritional index predict survival in patients with non-small cell lung cancer. World J Surg Oncol 13: 291, 2015.

32. Takahashi Y, Horio H, Hato T, Harada M, Matsutani N, Morita S and Kawamura M: Prognostic significance of preoperative Neutrophil-Lymphocyte ratios in patients with Stage I non-small cell lung cancer after complete resection. Ann Surg Oncol 22 (Suppl 3): S1324-S1331, 2015.

33. Zhang H, Zhang L, Zhu K, Shi B, Yin Y, Zhu J, Yue D, Zhang B and Wang C: Prognostic significance of combination of preoperative platelet count and Neutrophil-Lymphocyte ratio (COP-NLR) in patients with non-small cell lung cancer: Based on a large cohort study. PLoS One 10: e0126496, 2015.

34. Berardi R, Rinaldi S, Santoni M, Newsom-Davis T, Tiberi M, Morgese F, Caramanti M, Savini A, Ferrini C, Torniai M, et al: Prognostic models to predict survival in patients with advanced non-small cell lung cancer treated with first-line chemo- or targeted therapy. Oncotarget 7: 26916-26924, 2016.

35. Yin Y, Wang J, Wang X, Gu L, Pei H, Kuai S, Zhang Y and Shang Z: Prognostic value of the neutrophil to lymphocyte ratio in lung cancer: A meta-analysis. Clinics (Sao Paulo) 70: 524-530, 2015.

36. Sethi G, Shanmugam MK, Ramachandran L, Kumar AP and Tergaonkar V: Multifaceted link between cancer and inflammation. Biosci Rep 32: 1-15, 2012

37. Candido J and Hagemann T: Cancer-related inflammation. J Clin Immunol 33 (Suppl 1): S79-S84, 2013.

38. Kumar V and Sharma A: Neutrophils: Cinderella of innate immune system. Int Immunopharmacol 10: 1325-1334, 2010.
39. Borregaard N: Neutrophils, from marrow to microbes. Immunity 33: 657-670, 2010.

40. Proctor MJ, Morrison DS, Talwar D, Balmer SM, Fletcher CD, O'Reilly DS, Foulis AK, Horgan PG and McMillan DC: A comparison of inflammation-based prognostic scores in patients with cancer. A Glasgow Inflammation Outcome Study. Eur J Cancer 47: 2633-2641, 2011

41. Yu P and Fu YX: Tumor-infiltrating T lymphocytes: Friends or foes? Lab Invest 86: 231-245, 2006.

42. Kinoshita T, Muramatsu R, Fujita T, Nagumo H, Sakurai T, Noji S, Takahata E, Yaguchi T, Tsukamoto N, Kudo-Saito C, et al: Prognostic value of tumor-infiltrating lymphocytes differs depending on histological type and smoking habit in completely resected non-small-cell lung cancer. Ann Oncol 27: 2117-2123, 2016.

43. Hanahan D and Weinberg RA: Hallmarks of cancer: The next generation. Cell 144: 646-674, 2011

44. Inoue M, Okumura M, Sawabata N, Miyaoka E, Asamura H, Yoshino I, Tada H, Fujii Y, Nakanishi Y, Eguchi K, et al: Clinicopathological characteristics and surgical results of lung cancer patients aged up to 50 years: The Japanese lung cancer registry study 2004. Lung Cancer 83: 246-251, 2014.

45. Parsons A, Daley A, Begh R and Aveyard P: Influence of smoking cessation after diagnosis of early stage lung cancer on prognosis: Systematic review of observational studies with meta-analysis. BMJ 340: b5569, 2010.

46. Kanarek NF, Hooker CM, Mathieu L, Tsai HL, Rudin CM, Herman JG and Brock MV: Survival after community diagnosis of early-stage non-small cell lung cancer. Am J Med 127: 443-449, 2014

47. Mak RH, Hermann G, Lewis JH, Baldini EH, Chen AB, Colson YL, Hacker FH, Kozono D, Wee JO, Chen YH, et al: Outcomes by tumor histology and KRAS mutation status after lung stereotactic body radiation therapy for early-stage non-small-cell lung cancer. Clin Lung Cancer 16: 24-32, 2015.

48. Trani L, Myerson J, Ashley S, Young K, Sheri A, Hubner R, Puglisi M, Popat S and O'Brien ME: Histology classification is not a predictor of clinical outcomes in advanced non-small cell lung cancer (NSCLC) treated with vinorelbine or gemcitabine combinations. Lung Cancer 70: 200-204, 2010.

49. Yodying H, Matsuda A, Miyashita M, Matsumoto S, Sakurazawa N, Yamada M and Uchida E: Prognostic significance of Neutrophil-to-Lymphocyte ratio and platelet-to-Lymphocyte ratio in oncologic outcomes of esophageal cancer: A systematic review and meta-analysis. Ann Surg Oncol 23: 646-654, 2016.

50. Wei B, Yao M, Xing C, Wang W, Yao J, Hong Y, Liu Y and Fu P: The neutrophil lymphocyte ratio is associated with breast cancer prognosis: An updated systematic review and meta-analysis. Onco Targets Ther 9: 5567-5575, 2016

51. Sun J, Chen X, Gao P, Song Y, Huang X, Yang Y, Zhao J, Ma B, Gao X and Wang Z: Can the neutrophil to lymphocyte ratio be used to determine gastric cancer treatment outcomes? A systematic review and meta-analysis. Dis Markers 2016: 7862469, 2016

52. Sun XD, Shi XJ, Chen YG, Wang CL, Ma Q and Lv GY: Elevated preoperative Neutrophil-Lymphocyte ratio is associated with poor prognosis in hepatocellular carcinoma patients treated with liver transplantation: A meta-analysis. Gastroenterol Res Pract 2016: 4743808, 2016.

53. Yang JJ, Hu ZG, Shi WX, Deng T, He SQ and Yuan SG: Prognostic significance of neutrophil to lymphocyte ratio in pancreatic cancer: A meta-analysis. World J Gastroenterol 21: 2807-2815, 2015.

54. Tsai PL, Su WJ, Leung WH, Lai CT and Liu CK Neutrophil-lymphocyte ratio and CEA level as prognostic and predictive factors in colorectal cancer: A systematic review and meta-analysis. J Cancer Res Ther 12: 582-589, 2016.

55. Hu K, Lou L, Ye J and Zhang S: Prognostic role of the neutrophil-lymphocyte ratio in renal cell carcinoma: A meta-analysis. BMJ Open 5: e006404, 2015

56. Tang L, Li X, Wang B, Luo G, Gu L, Chen L, Liu K, Gao Y and Zhang X: Prognostic value of Neutrophil-to-Lymphocyte ratio in localized and advanced prostate cancer: A systematic review and meta-analysis. PLoS One 11: e0153981, 2016.

57. Davis JL, Moutinho V Jr, Panageas KS and Coit DG: A peripheral blood biomarker estimates probability of survival: The neutrophil-lymphocyte ratio in noncancer patients. Biomark Med 10: 953-957, 2016. 